\title{
Condutas para reparação da cavidade anoftálmica no Brasil
}

\section{Trends on anophthalmic socket repair in Brazil}

Roberta lilian Fernandes de Sousa ${ }^{1}$, Silvana Artiol Schellini² ${ }^{2}$ Denise de Cássia Moreira Zornoff ${ }^{3}$, Carlos Roberto Padovani ${ }^{4}$

\section{RESUMO}

Objetivos: Avaliar as condutas mais utilizadas no tratamento da cavidade anoftálmica no Brasil, comparando-as com a realidade mundial.

Métodos: Estudo exploratório, usando questionário eletrônico enviado pela Internet para oftalmologistas membros da Sociedade Brasileira de Cirurgia Plástica Ocular, Vias Lacrimais e Órbita - SBCPO. As respostas obtidas foram avaliadas por meio de análise de aderência, utilizando o teste do Qui-quadrado.

Resultados: Foram recebidos 75 questionários respondidos. Cinquenta e três por cento dos entrevistados tratam cavidade anoftálmica frequentemente e o implante de esfera de polimetilmatacrilato, de $18 \mathrm{~mm}$ de diâmetro, é o usado pelos entrevistados na maioria das cirurgias, sendo revestido principalmente com esclera (92\%). Apenas sete entrevistados já utilizaram implante acoplado com prótese externa. Oitenta e dois por cento dos entrevistados usam a técnica do enxerto dermoadiposo. O acompanhamento destes pacientes é feito semestralmente pela maior parte dos entrevistados.

Conclusão: $O$ tratamento da cavidade anoftálmica no Brasil geralmente é feito usando a esfera de polimetilmetacrilato, de diâmetro 18 milímetros. Implantes acoplados dificilmente são usados.

Descritores: Anoftalmia; Olho artificial; Órbita; Evisceração do olho; Brasil

\section{ABSTRACT}

Purpose: To determine the most common approach to repair the anophthalmic socket in Brazil, and to compare the data with the trends in other countries.

Methods: Exploratory study using electronic questionnaire sent by Internet to ophthalmologists members of the Brazilian Orbit and Oculoplastic Society (SBCPO). The received answers were analyzed by adhesion analysis, using Chi-square test.

Results: We received 75 answered questionnaires. Fifty-threepercent of the respondents frequently treat anophthalmic socket and use the $18 \mathrm{~mm}$ diameter polymethylmethacrylate sphere in the majority of the surgeries, mainly covered by sclera (92\%). Only seven interviewees had used integrated implants with pegging procedure. Eighty-two per cent of the ophthalmologists use the dermolipid graft to reconstruct the anophthalmic socket. They also follow the patients bi-annually.

Conclusions: The treatment of anophthalmic socket in Brazil generally involve polymethylmethacrylate sphere with $18 \mathrm{~mm}$ diameter. Pegging procedure is uncommon between us.

Keywords: Anophthalmos; Eye artificial; Orbit; Eye evisceration; Brazil

\section{INTRODUÇÃO}

O tratamento e cuidado na reconstrução da cavidade anoftálmica são preocupações humanas desde tempos muito antigos. O uso de próteses oculares, por exemplo, é descrito desde os tempos egíp$\operatorname{cios}^{(1)}$, quando uma prótese de metal com o desenho de um olho era usada para esconder o defeito facial.

O emprego das cirurgias de evisceração e enucleação como tratamento final de algumas alterações oculares foi iniciado apenas na metade do século XIX ${ }^{(1)}$. E quase que concomitantemente ao desenvolvimento das técnicas para enucleação e evisceração, ocorreu a evolução de materiais a serem utilizados para reparação da cavidade anoftálmica. Vários tipos de implantes já foram testados, entre eles: vidro, polietileno poroso, silicone, hidroxiapatita natural e sintética e polimetilmetacrilato (PMMA) ${ }^{(1)}$.

No Brasil, ainda próximo dos anos 80 do século passado, apesar do grande desenvolvimento no tratamento da cavidade anoftálmica ocorrido em outros países, era muito comum que após as enucleações e eviscerações o paciente evoluísse a sua própria sorte, sem a utilização de implantes orbitários, utilizando ou não as próteses externas.

Atualmente, o implante de PMMA parece ser o mais utilizado no Brasil. Entretanto, não há dados concretos para confirmar esta suposição.

Este estudo procura avaliar quais as condutas mais utilizadas no tratamento da cavidade anoftálmica no Brasil, possibilitando o conhecimento da realidade brasileira diante desta afecção, assim como compará-la à realidade mundial no tratamento da cavidade anoftálmica.

\section{MÉTODOS}

O presente estudo foi avaliado e aprovado para execução pelo Comitê de Ética em Pesquisa da Faculdade de Medicina de Botucatu.

Submetido para publicação: 4 de Novembro de 2011

Aceito para publicação: 9 de Outubro de 2012

Trabalho realizado na Universidade Estadual Paulista "Júlio de Mesquita Filho" - UNESP - Botucatu (SP), Brasil.

Médica, Departamento de Oftalmologia, Otorrinolaringologia e Cirurgia de Cabeça e Pescoço, Faculdade de Medicina de Botucatu, Universidade Estadual Paulista "Júlio de Mesquita Filho" UNESP - Botucatu (SP), Brasil.

Professora, Departamento de Oftalmologia, Otorrinolaringologia e Cirurgia de Cabeça e Pescoço da Faculdade de Medicina de Botucatu, Universidade Estadual Paulista "Júlio de Mesquita Filho" UNESP - Botucatu (SP), Brasil.

Núcleo de Ensino a Distância, Faculdade de Medicina de Botucatu, Universidade Estadual Paulista ("Júlio de Mesquita Filho" - UNESP - Botucatu (SP), Brasil.

Professor, Departamento de Estatística, Instituto de Biociências, Universidade Estadual Paulista

"Júlio de Mesquita Filho" - UNESP - Botucatu (SP), Brasil.

Financiamento: Projeto financiado pela FAPESP.

Divulgação de potenciais conflitos de interesse: R.L.F.de Sousa, Nenhum; S.A.Schellini, Nenhum, D.C.M.Zornoff, Nenhum; C.R.Padovani, Nenhum.

Endereço para correspondência: Roberta Lilian Fernandes de Sousa. Rua Marília, 427 - Apto. 3 Botucatu (SP) - 18608-560 - Brasil - E-mail: rlfsousa@yahoo.com.br

Aprovado pelo Comitê de Ética em Pesquisa da Faculdade de Medicina de Botucatu. Protocolo no 3176-2009. 
Foi realizada pesquisa prospectiva por meio de questionário eletrônico enviado pela Internet para oftalmologistas que são membros da Sociedade Brasileira de Cirurgia Plástica Ocular, Vias Lacrimais e Órbita (SBCPO), que são as pessoas que mais trabalham com portadores de cavidade anoftálmica em nosso país. O questionário também foi postado no site da SBCPO, permitindo o seu preenchimento também por este meio.

O questionário (anexo) era composto de 12 questões de múltipla escolha e abordava quais eram as condutas dos médicos oftalmolo-

\section{Anexo. Questionário: condutas em cavidade anoftálmica}

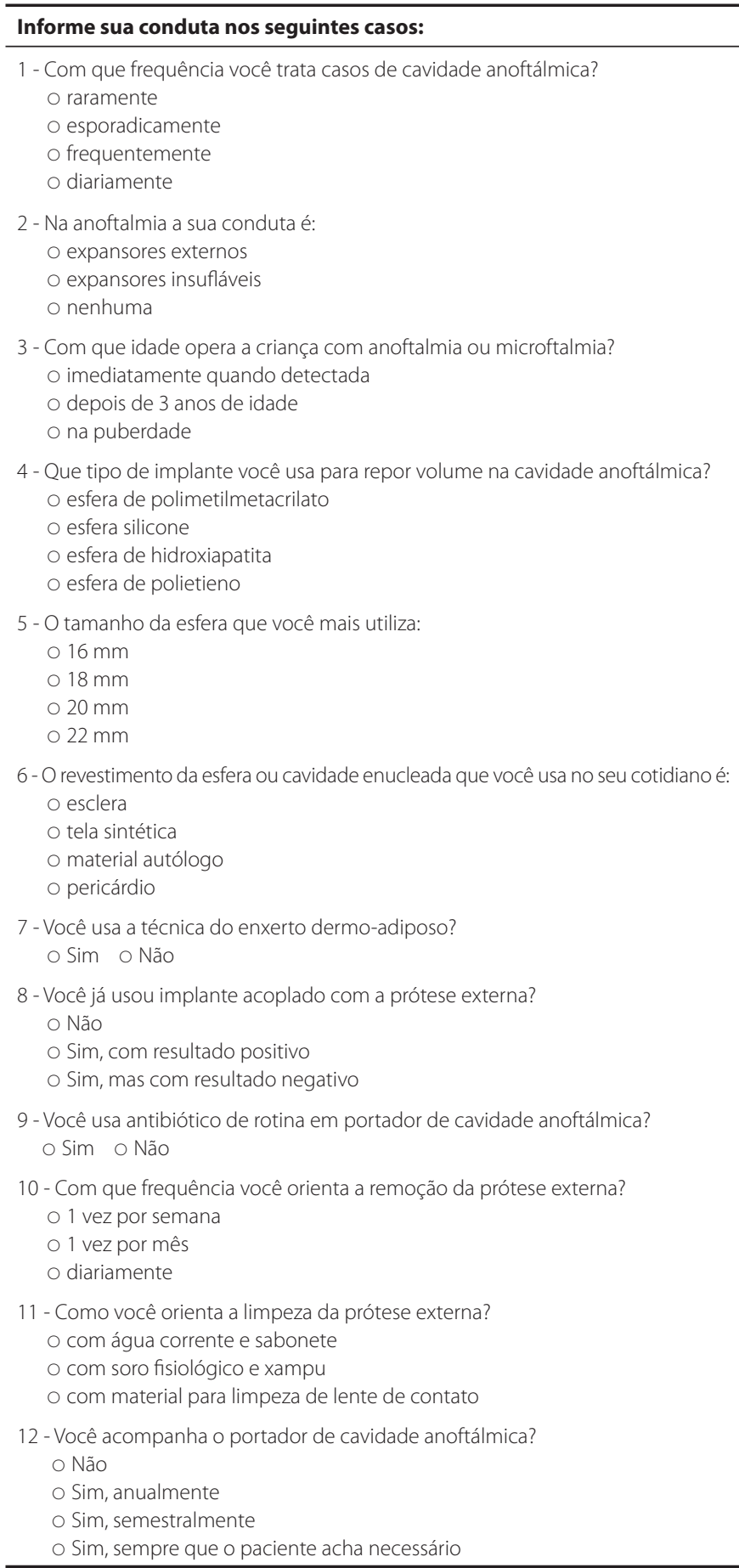

gistas diante das cirurgias realizadas para o tratamento da cavidade, a sua reconstrução e o tratamento empregado no pós-operatório dos pacientes portadores de cavidade anoftálmica.

As respostas obtidas foram transferidas para tabela Excel e avaliadas por meio de análise de aderência, utilizando o teste do Qui-quadrado, considerando segundo a distribuição dos dados equiprováveis e o nível de significância de 5\%.

\section{RESULTADOS}

Foram enviados via on-line os questionários para os 249 oftalmologistas membros da SBCPO. Foram respondidos 75 questionários, o que corresponde a $30,12 \%$ do total de oftalmologistas que participaram da pesquisa.

Aproximadamente $53 \%$ dos entrevistados tratam cavidade anoftálmica frequentemente, e sete entrevistados tratam diariamente.

Oitenta e dois por cento dos entrevistados tratam a anoftalmia com o uso de expansores externos. Disseram, também, que submetem as crianças à cirurgia depois de 3 anos de idade, 48 entrevistados (64\%). Dezessete oftalmologistas (22,7\%) submetem as crianças à cirurgia imediatamente e apenas $8(10,7 \%)$, na puberdade (Figura 1).

O implante de cavidade mais utilizado pelos oftalmologistas da SBCPO é o de PMMA, correspondendo à preferência de $62,7 \%$ dos entrevistados. O implante de polietileno é a escolha de 20\% dos sócios, seguido do implante de hidroxiapatita (8\%) e de silicone (4\%) (Figura 2).

Em relação ao tamanho da esfera, o mais utilizado é o de $18 \mathrm{~mm}$ (60\%), seguido da esfera de 16 mm (29,3\%) e de 20 mm (8\%). Nenhum dos entrevistados referiu o uso de esferas de $22 \mathrm{~mm}$ (Figura 3).

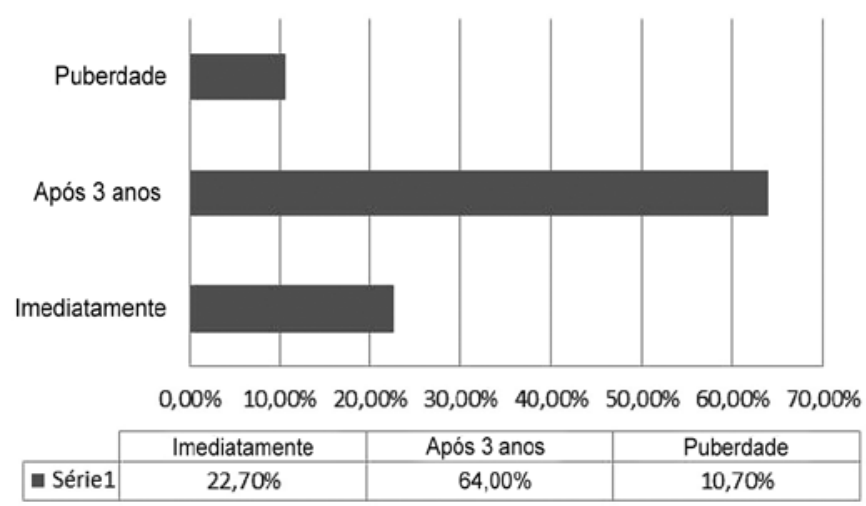

Figura 1. Idade de tratamento da anoftalmia congênita referida pelos oftalmologistas da SBCPO - UNESP, 2011.

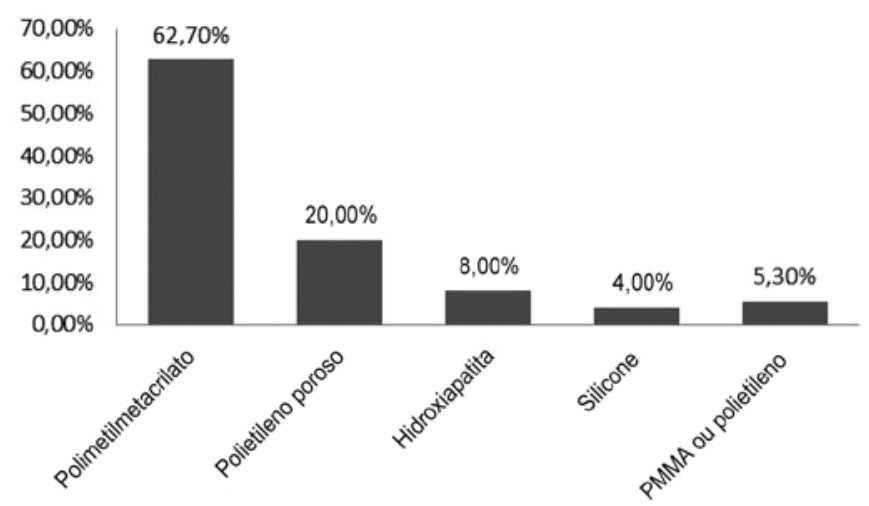

Figura 2. Distribuição do tipo de esfera utilizada para tratamento da cavidade anoftálmica pelos oftalmologistas da SBCPO - UNESP, 2011. 
Quanto ao revestimento da esfera, 92\% (69) dos entrevistados fazem uso de esclera e quatro entrevistados (5,3\%) fazem uso de material autólogo para revestí-la.

Quase 82\% dos oftalmologistas usam a técnica do enxerto dermoadiposo para reconstrução da cavidade anoftálmica.

O uso de implante acoplado à prótese externa foi relatado por sete entrevistados, sendo que quatro deles relataram ter tido um resultado positivo com a aplicação desta técnica. Os demais entrevistados (68 - 90,7\%) relataram nunca terem utilizado o implante acoplado à prótese externa.

Não houve diferença com significância estatística em relação ao uso de antibióticos de rotina pelo portador de cavidade anoftálmica: 52\% não prescrevem e 48\% prescrevem rotineiramente antibióticos para seus pacientes.

Quanto aos cuidados pós-operatórios, também não houve diferença estatística significativa entre os entrevistados que orientam a limpeza semanal ou diária da prótese externa (48\% e 40\% respectivamente). Apenas oito oftalmologistas orientam seus pacientes a realizarem limpeza mensal da prótese externa. Além disso, a limpeza é orientada a ser feita com água e sabonete por 71,6\% dos entrevistados, seguido de soro fisiológico e xampu por 14,9\% e material para limpeza de lente de contato por 12,2\%.

Dois entrevistados $(2,66 \%)$ responderam que não acompanham os pacientes portadores de cavidade anoftálmica. Trinta e seis por cento fazem o acompanhamento anualmente, 42,7\%, semestralmente; $14,7 \%$, sempre que o paciente achar necessário. Um dos oftalmologistas acompanha seus pacientes anualmente ou sempre que o

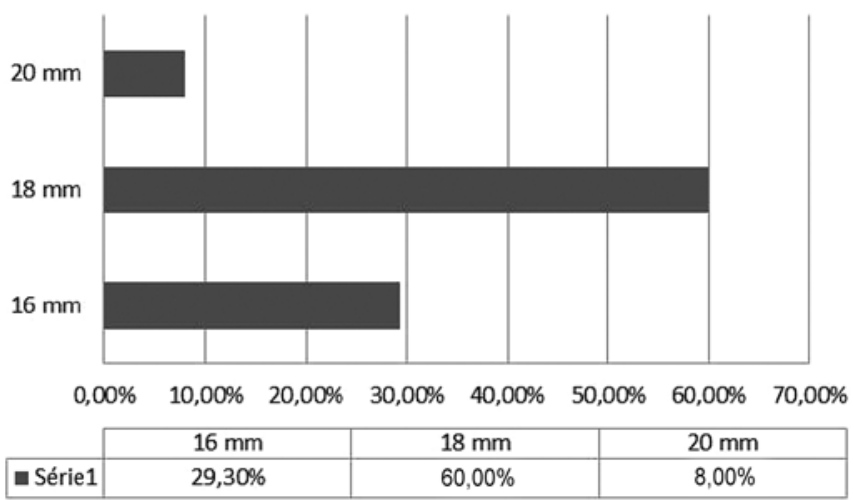

Figura 3. Distribuição do tamanho da esfera utilizada no reparo da cavidade anoftálmica pelos oftalmologistas da SBCPO - UNESP, 2011. paciente achar necessário e outro, semestralmente ou sempre que o paciente achar necessário (Figura 4).

\section{DISCUSSÃO}

Estudos baseados em questionários podem ter fatores complicadores, como a taxa de adesão de respostas. Neste sentido, no presente estudo, a porcentagem de adesão ao questionário foi baixa $(30,12 \%)$, quando comparado com estudos semelhantes feitos nos Estados Unidos, onde 60\% retornaram as respostas ${ }^{(2)}$, e no Reino Unido, onde foi possível obter $51 \%$ de respostas ${ }^{(3)}$. Outro estudo realizado nos Estados Unidos, porém, mostrou taxa de adesão de 31,4\%, bem próxima da encontrada em nosso levantamento ${ }^{(4)}$.

Outro importante fator a ser considerado nos estudos baseados em questionários é o grau de confiabilidade nas respostas. É importante lembrar, que a validade do estudo é totalmente dependente da veracidade das respostas recebidas. Com relação a este ponto, aplicar o questionário para grupos específicos de pessoas, como o que foi praticado, pode reduzir o viés.

A frequência de tratamento da cavidade anoftálmica mostrou-se alta em nosso estudo. No Reino Unido, no ano de 2006, foram enviados os questionários para todos os oftalmologistas do país, revelando que 35\% dos médicos não tratavam cavidade anoftálmica, apesar da maioria das cirurgias ter sido realizada por profissionais com subespecialização em órbita, plástica ocular e vias lacrimais daquele país. No caso do presente estudo, a alta porcentagem de oftalmologistas que trata frequentemente portadores de cavidade anoftálmica foi alta devido ao fato de termos considerado os membros da SBCPO como os participantes da pesquisa. Sem dúvida, oftalmologistas gerais não se ocupam com a mesma intensidade destes pacientes.

Não foi questionado qual o tipo de cirurgia mais realizado pelos oftalmologistas, se evisceração ou enucleação. Entretanto, levantamento feito em nosso serviço mostrou que, na década entre 1980 e 1990, quando se compara com a década anterior (1970 até 1980), a evisceração passou a ser mais executada que a enucleação, que era a cirurgia preferida dos anos $70^{(5)}$. Outro estudo, também realizado em nosso serviço no ano de 2000, confirmou esta mudança ${ }^{(6)}$. Esta é uma tendência mundial, talvez devido às melhores condições de movimentação da esfera no pós-operatório destes pacientes, melhorando a estética dos mesmos, ou talvez devido ao menor trauma cirúrgico ou até mesmo devido ao implante utilizado, já que os norte-americanos enucleiam mais que evisceram, muito provavelmente devido ao tipo de reconstrução que eles empregam, centrada no uso de implantes integrados ${ }^{(4)}$.

Desde a segunda Grande Guerra, os implantes confeccionados de silicone e de PMMA, implantes estes de superfície lisa, chamados de não integrados, dominavam o mercado do mundo todo para a reparação da cavidade anoftálmica(1).

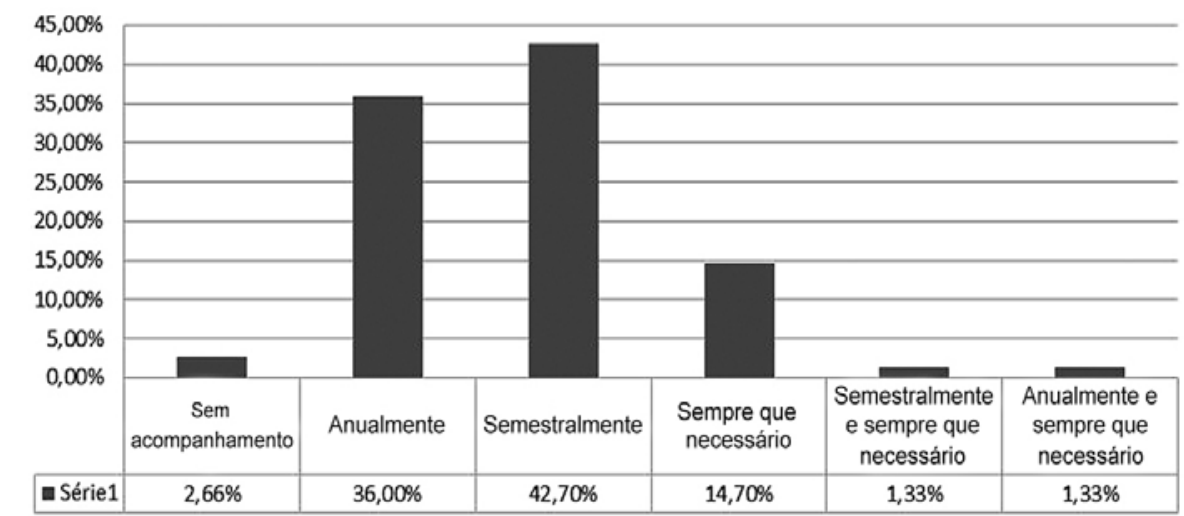

Figura 4. Conduta dos oftalmologistas da SBCPO adotada para acompanhamento dos portadores de cavidade anoftálmica - UNESP, b2011. 
A partir do ano de 1987, o panorama se alterou sobremaneira, passando a terem importância os implantes integrados, confeccionados de hidroxiapatita natural, tendo sido o novo implante sugerido por Perry ${ }^{(7)}$.

Surgiram também as hidroxiapatitas sintéticas, usadas inclusive aqui no Brasil(8,9). Outro tipo de biomaterial, o polietileno poroso, passou a ser disponível para uso em cavidade anoftálmica desde meados de $1991^{(10)}$.

O presente estudo confirmou o que já era suspeição antes do início desta pesquisa, quando aponta que, atualmente, no Brasil, ainda o implante mais utilizado é o de PMMA, diferentemente de outros países, como os Estados Unidos, no qual o implante mais utilizado é o polietileno poroso ${ }^{(4)}$. As esferas de acrílico foram o segundo implante mais usado no Reino Unido, onde o polietileno poroso é, também, o implante mais usado(3).

A técnica do enxerto dermoadiposo é bastante difundida entre nós, sendo utilizada por cerca de $80 \%$ dos entrevistados. Sem dúvida, esta é uma boa opção, em especial quando não se pode contar com os implantes aloplásticos.

O uso de esclera doadora como envoltório dos implantes de cavidade é também muito comum nos Estados Unidos. No Reino Unido, entretanto, o uso de malha sintética é preferido pela maioria dos cirurgiões ${ }^{(3)}$.

Uma importante semelhança diz respeito ao uso de implantes acoplados à prótese externa. Os "pegs", considerados inicialmente como uma das maiores vantagens do uso dos implantes integrados pela possibilidade de acoplagem dos implantes com as próteses externas, levaram a muitas complicações ${ }^{(11)}$. Em outros países onde estudos semelhantes foram realizados, o uso deste tipo de implante também é pequeno, embora seja relatado sucesso do uso da acoplagem dos implantes com a prótese externa, em especial quando se utiliza pinos de titânio ${ }^{(12,13)}$.

Os cuidados pós-operatórios dos pacientes portadores de cavidade anoftálmica também foram avaliados em estudo feito nos Estados Unidos, interessando a conduta que os oftalmologistas possuem diante do portador de cavidade anoftálmica. Praticamente todos os entrevistados, no estudo norte-americano, indicam a limpeza das próteses externas em água corrente e com algum sabão higienizador ${ }^{(14)}$. A antibioticoterapia pós-operatória era, também, realizada por todos os oftalmologistas entrevistados, variando, apenas, o número de dias de uso destes medicamentos ${ }^{(14)}$. Entretanto, o questionário utilizado no presente estudo não previa dados com os quais fosse possível comparar a rotina de acompanhamento destes pacientes.

\section{CONCLUSÃO}

O tratamento da cavidade anoftálmica é frequente entre os oftalmologistas membros da SBCPO. A esfera de PMMA é a mais utilizada pelos oftalmologistas brasileiros, principalmente no diâmetro de 18 milímetros. O uso de implante acoplado com a prótese externa é raro entre nós. A grande maioria dos cirurgiões está familiarizada com a técnica do enxerto dermoadiposo. O acompanhamento dos pacientes em geral é semestral, com orientação da limpeza da prótese externa com água e sabonete, semanalmente.

\section{REFERÊNCIAS}

1. den Tonkelaar I, Henkes HE, Leersum GK. Herman Snellen (1834-1908) and Müller's 'reform-auge'. A short history of the artificial eye. Doc Ophthalmol. 1991;77(4):349-54.

2. Hornblass A, Biesman BS, Eviatar JA. Current techniques of enucleation: a survey of 5,439 intraorbital implants and a review of the literature. Ophthal Plast Reconstr Surg. 1995;11(2):77-86; discussion 87-8.

3. Alwitry A, West S, King J, Foss AJ, Abercrombie LC. Long-term follow-up of porous polyethylene spherical implants after enucleation and evisceration. Ophthal Plast Reconstr Surg. 2007;23(1):11-5.

4. Su GW, Yen MT. Current trends in managing the anophthalmic socket after primary enucleation and evisceration. Ophthal Plast Reconstr Surg. 2004;20(4): 274-80.

5. Schellini SA, Oliveira DA, Oliveira CA, Hoyama E, Padovani CR. Evisceração e enucleação na Faculdade de Medicina de Botucatu - UNESP: comparação entre duas décadas. Salusvita. 2003;22(1):71-84.

6. Hoyama E, Schellini SA, Ferreira VL, Rossa R, Padovani CR. Uso de esferas de polietileno poroso em cavidade anoftálmica. Rev Bras Oftalmol. 2000;59(1):40-4.

7. Perry AC. Integrated orbital implants. Adv Ophthalmic Plast Reconstr Surg. 1988;8: 75- 81. Review.

8. Schellini SA, Hoyama E, Padovani CR, Ferreira VL, Rossa R. Complicações com uso de esferas não integráveis e integráveis na reconstrução da cavidade anoftálmica. Arq Bras Oftalmol. 2000;63(3):175-8.

9. Jordan DR, Hwang I, McEachren T, Brownstein S, Gilberg S, Grahovac S, et al. Brazilian hydroxyapatite implant. Ophthal Plast Reconstr Surg. 2000;16(5):363-9.

10. Karesh JW, Dresner SC. High-density porous polyethylene (Medpor) as a successful anophthalmic socket implant. Ophthalmology. 1994;101(10):1688-95; discussion 1695-6.

11. Shoamanesh A, Pang NK, Oestreicher JH. Complications of orbital implants: a review of 542 patients who have undergone orbital implantation and 275 subsequent PEG placements. Orbit. 2007;26(3):173-82.

12. Johnson RL, Ramstead CL, Nathoo N. Pegging the porous orbital implant. Ophthal Plast Reconstr Surg. 2011;27(2):74-5.

13. Yoon JS, Lew H, Kim SJ, Lee SY. Exposure rate of hydroxyapatite orbital implants a 15-year experience of 802 cases. Ophthalmology. 2008;115(3):566-72.e2. Comment in Ophthalmology. 2008;115(12):2320-1; author reply 2321. Ophthalmology. 2008; 115(11):2096; author reply 2096-7.

14. Osborn KL, Hettler D. A survey of recommendations on the care of ocular prostheses. Optometry. 2010;81(3):142-5. 